\title{
BIM-based Collaborative Management and Intelligent Manufacturing in the Shenzhong Link Project
}

\author{
Cheng Liu ${ }^{1 *}$, Qinghan $\mathrm{Bu}^{2}$, Shutao $\mathrm{Lin}^{2}$, and Faxiong $\mathrm{Li}^{2}$ \\ ${ }^{1}$ Research Institute of Highway (RIOH), Ministry of Transport, Beijing, 100088, China \\ ${ }^{2}$ China-Road Transportation Verification \& Inspection Hi-Tech Co., Ltd., Beijing, 100088, China
}

\begin{abstract}
Building Information Modelling (BIM) technology has become a central topic in infrastructure construction industry recently in China. This paper describes the applications of BIM technology, including 3D digital design, intelligent manufacturing, smart site and BIM-based collaborative management platform, in a mega infrastructure project, the Shenzhong Link. Through BIM technology, Shenzhong Link strives to achieve the goal of "improving quality, increasing efficiency, reducing costs and achieve traceability"
\end{abstract}

\section{Introduction}

After years of engineering applications, Building Information Modelling (BIM) goes beyond the production of 3D models and become a comprehensive information technology involving 3D model application, project collaborative management, smart site, intelligent construction, etc[1]. Infrastructure projects in general have to handle the highly complex and diverse nature of project requirements, particularly for managing the quality, safety, schedule and expenses during the construction[2]. BIM therefore, provides an emerging new paradigm for construction management in infrastructure construction. In China, the application of BIM has become a central topic to improve the productivity and collaborative level in infrastructure construction industry recently[3,4]. This paper aims to describe the applications of BIM-based collaborative management and intelligent construction in a mega infrastructure project, the Shenzhong Link.

\section{Overview of BIM application in Shenzhong Link}

The Shenzhong Link Project connects Shenzheng city and Zhongshan city and is located in the core area of Guangdong, Hong Kong and Macao Bay Area. It is a world-class cluster project integrating "bridge, island, tunnel and underwater interchange". It is located $30 \mathrm{~km}$ away from Humen Bridge in the north and $38 \mathrm{~km}$ away from Hong Kong-Zhuhai-Macao Bridge in the south. This project adopts an East Tunnel West Bridge layout. The total length of the route is about $24 \mathrm{~km}$, including a 1666-meter-span suspension bridge (Lingting Bridge), a 6845-meter immersed tunnel (Fanshi Tunnel), two artificial islands, and three interchanges. This project follows a $100 \mathrm{~km} / \mathrm{h}$, two-way, 8-lane highway technical standard. The total project budget is about 44.69 billion yuan. 


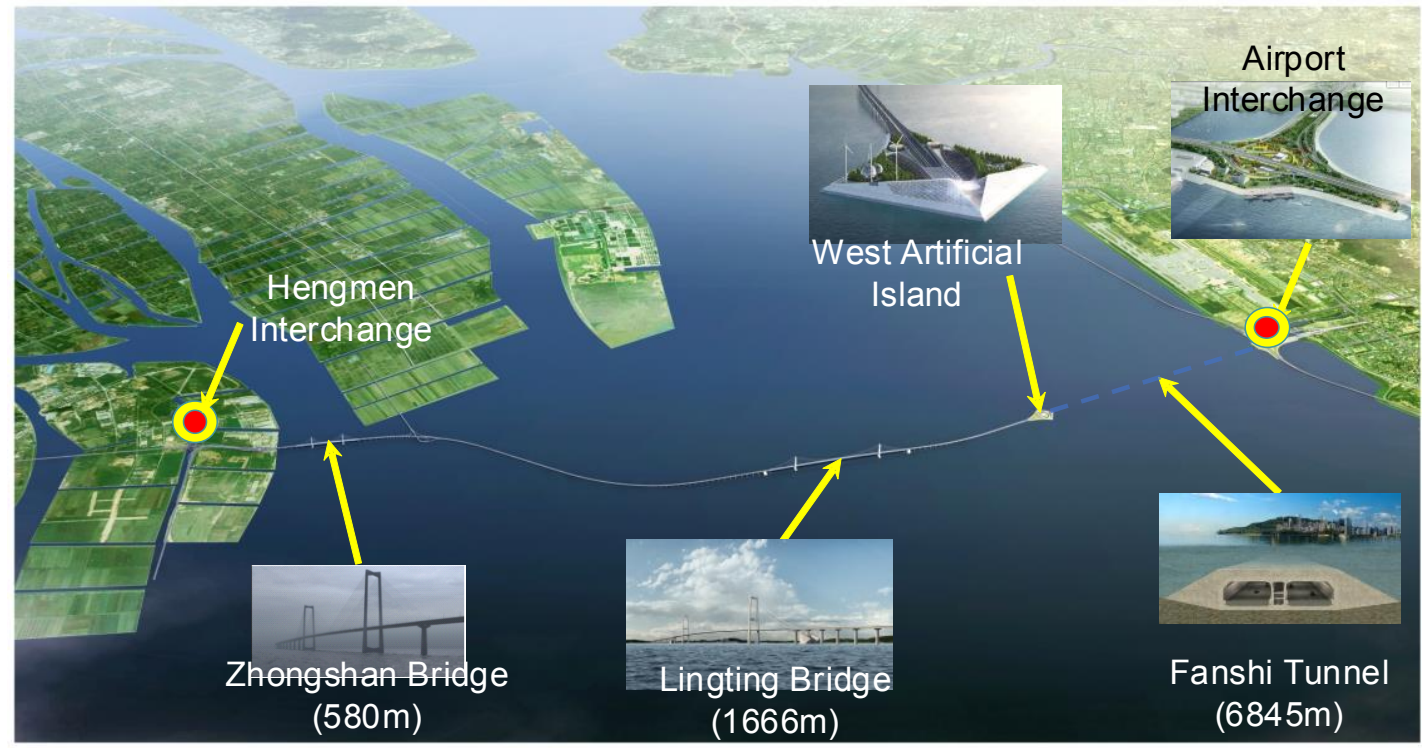

Figure 1 Engineering overview of the Shenzhong Link

As a world-class cross-sea cluster project, the Shenzhong Link has numerous participants. It places high demands on digital design level, refined management level and automated manufacturing level. To support the construction goal of "World-class 100year Cross-Sea Tunnel Project", Shenzhong Link proposed to build a "Digital Shenzhong, Smart Shenzhong" demonstration project with a full life cycle and continuously improve the level of digitalization, informationization, automation and intelligence of construction progress through BIM technology.

According to different implementation stages, BIM application during construction period is divided into three stages: pre-construction preparation stage, construction stage, and completion stage. The preconstruction preparation stage involves working mechanism and resource allocation. The construction stage includes four aspects: digital design, intelligent manufacturing, collaborative management and smart site. The completion stage includes three aspects: acceptance of the completion model, acceptance of technical results and acceptance of the project data.

The application of BIM technology in the construction stage is divided into two main lines: 1) BIM modeling and model application, which make full use of the visibility, computability and interactivity features of BIM technology to improve the design, construction and management level; 2): BIM-based application, which adopts BIM model as a carrier to integrate data of various stages of the project, including intelligent manufacturing, BIM-based collaborative management platform and smart site system.

\section{3D Digital Design}

Three-dimensional digital design and parametric tools are adopted in all majors in the Shenzhong Link. Through the collision inspection function of BIM technology, the position conflicts of complex structural parts such as island-tunnel joint, anchorage zone and cable-tower joint are automatically detected, and the problems of "wrong, leaking, bumping and missing" of the design drawings are solved in advance. The advantages of BIM design tool are fully used in drawing checking, deep design, site planning, construction stage simulation, which reduces the engineering changes and optimizes the resource allocation.

The BIM modeling and delivery standards are developed. BIM models are transformed into standard data formats, and submitted to the unified model platform for further use including steel shell manufacturing, girder construction, smart site, etc. 


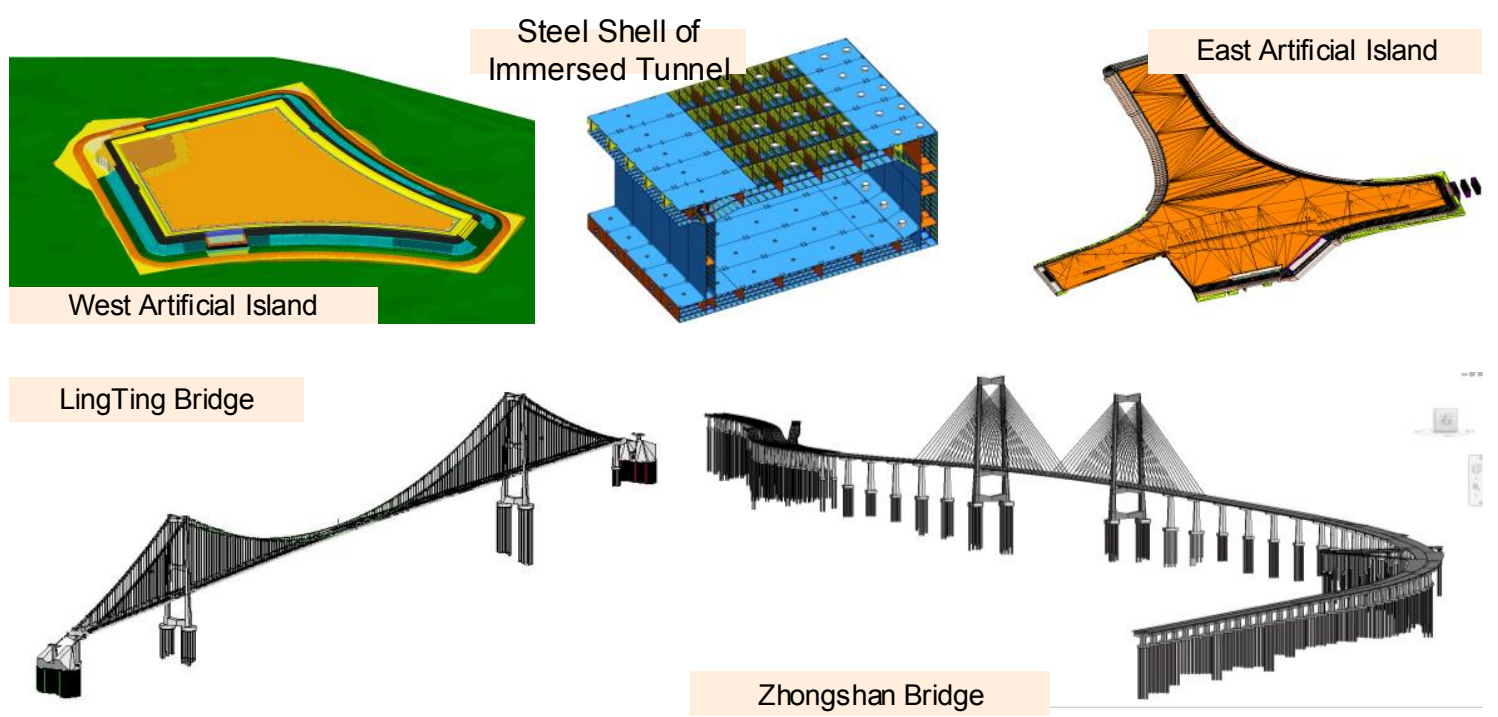

Figure 2 BIM modeling of Shenzhong Link

\section{Intelligent Manufacturing}

The intelligent manufacturing production line for the steel shells of immersed tunnel is built. The digital production design results of the immersed steel shell are transmitted to the intelligent manufacturing execution system (MES system). After receiving the production instructions, the MES system performs intelligent scheduling. The steel shells are manufactured in the workshop through the $\mathrm{CNC}$ automatic cutting pipeline, the plate welding pipeline, the block automatic welding

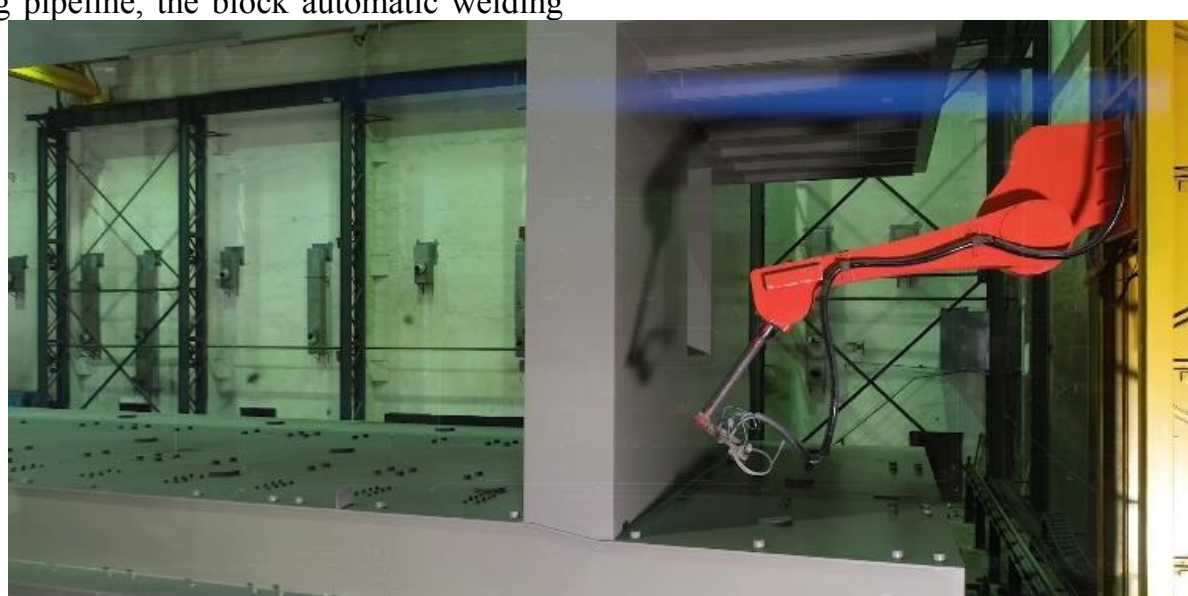

Figure 3 Automatic painting production line of the steel shells of the immersed tunnel

An intelligent processing line for rebars is also established. Rebars are first cut in a CNC rebar cutting line, and then bent in a CNC bending line, and finally assembled according to the design data transmitted from the BIM platform. A rebar centralized distribution management system realizes the information management of the whole process such as material storage, rebar processing, semi-finished product storage, rebar cage production, and on-site rebar cage installation through QR code and RFID technology.

prefabricated beam production line, and the automatic painting pipeline. Internet of Things (IoT) of plants is adopted to collect materials, equipment, and process data in real time. A three-dimensional visualized weld map is established for all the welds of the steel shell structure, and hence the whole process of the weld quality is traced. A smart pouring trolley is developed. The pouring trolley automatically positions the pouring hole according to the programmed coordinates, and closes the valve and lifts the lowering pipe according to the real-time feedback of the liquid level finder.
An intelligent girder factory is integrated. Each girder is built through the rebar numerical control machining, concrete intelligent mixing, formwork automatic control, automatic prestressing, automatic grouting, and intelligent spray maintenance. An IoT management platform is developed for the girder factory, integrating the BIM model, the IoT technology, the automatic positioning and the video monitoring technology to realize production process visualization, material tracking, production planning and scheduling, and quality tracing. 


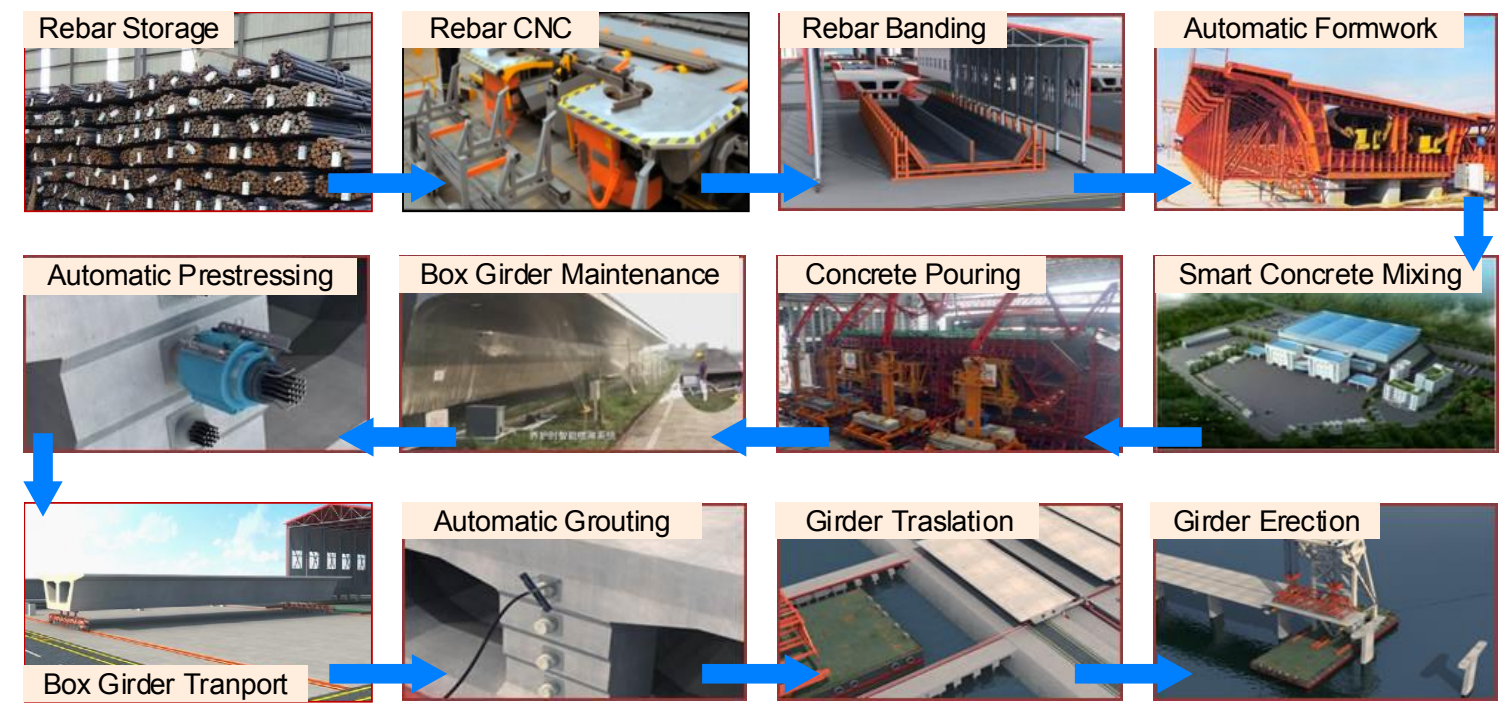

Figure 4 Production pipeline in the intelligent girder factory

\section{Collaborate Management}

The Shenzhong Link has a large scale and a large number of participating parties. It is very important to build a project collaborative management platform with full participation, data sharing and information interconnection. The collaborative management platform developed is supported by multi-terminal: data processing and file transfer on the web side; BIM model loading and visual control on the PC side; on-site mobile management at the mobile terminal service engineering site. The collaborative management platform uses the BIM model as the carrier to associate the engineering basic data with the unit partial code. The inspection process from the mobile terminal can feedback the actual project progress in real time to realize a real-time project schedule management. On-site quality inspection and

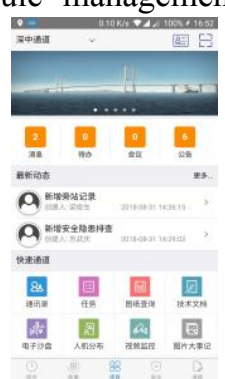

手机APP

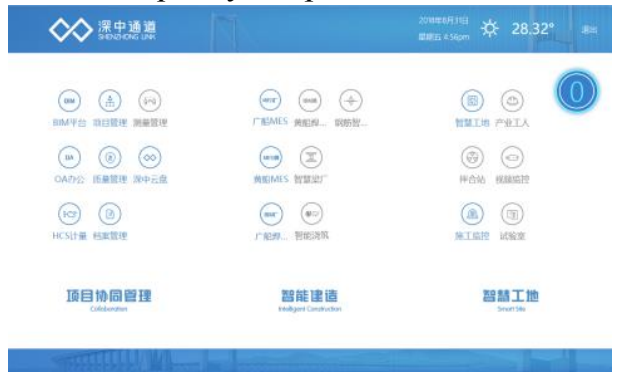

网页端 process inspection are carried out simultaneously, and engineering quality assurance documents are generated in the quality management system. Metering payment system are supported, where the schedule and quality module automatically generate the current engineering quantity measurement list to assist the payment. The data generated by each major business module is associated with the BIM model by unique codes.

Shenzhong Link also promoted digital signature technology to realize paperless and automatic filing of approval forms, quality inspection forms, and measurement documents. The Shenzhong Link has been listed as a "single-set" pilot project for the archive management of the Chinese National Department of Archives.

Figure 5 APP, web and PC terminals of the collaborate management platform (icons in Chinese)

\section{Smart Site}

The essence of the smart site is based on the IoT application. It collects, summarizes, analyses and predicts the information of the construction site in real time, and thus solves the difficulty of remote monitoring and supervision.

The smart site of the Shenzhong Link uses the BIMbased collaborative management platform as the carrier to connect people, vehicles, ships, special equipment, concrete mixing stations, field laboratory through smart cards, equipment sensors, GPS locators, intelligent terminals, etc. The smart site application enables dynamic statistics, equipment trajectory and safety monitoring and warning, material tracking, laboratory monitoring, remote video monitoring, and emergency command.

After entering the market, the laborers will register the real-name system and issue a smart ID card. The smart ID card integrated with GPS technology can realize positioning and one-button alarming. Through the smart card and central access control system, the work 
dynamics, trajectory and safety status of the laborers can be abstained in real time.

After the equipment and construction vessels enter the site, they will also register with the real name system, and install the GPS locator, print the QR code of the equipment. Safety monitoring system is installed on special operation equipment, to collect real-time information such as displacement, inclination, strain and jack stroke to realize safety monitoring and early warning.

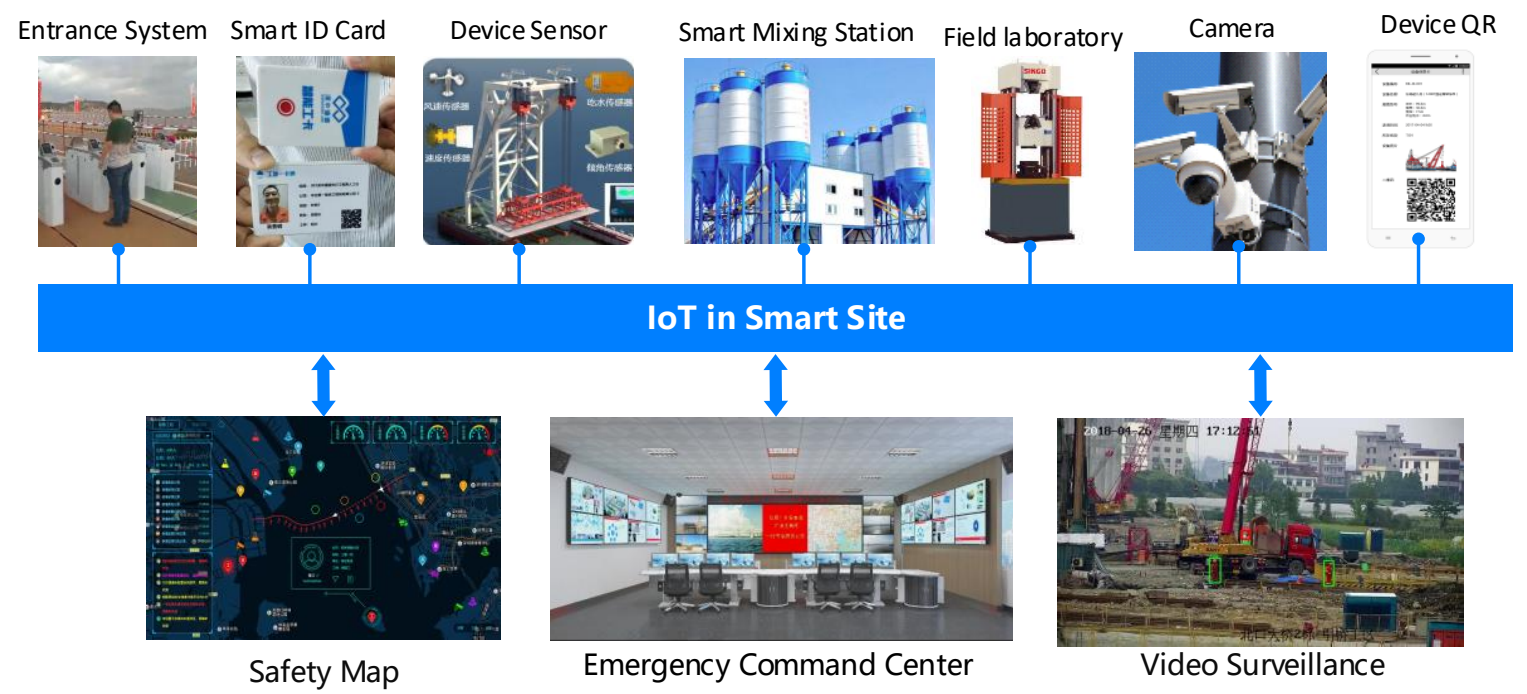

Figure 6 IoT in smart site in Shenzhong Link

\section{Conclusion}

BIM and intelligent manufacturing technology are the inevitable trend of the infrastructure construction industry transformation in the industrial $4.0 \mathrm{era}$, and the only way to transform and develop the transportation infrastructure field. At present, various BIM applications in the Shenzhong Link have shown strong application value, and are constantly deepening and iterating. The BIM application of Shenzhong Link will continue to adhere to the principle of "innovation-driven, problemoriented, details first", and effectively improve the quality and safety of the project, and strive to become a demonstration of BIM technology in the infrastructure industry.

\section{Acknowledgments}

The authors wish to thank the funding of Science and Technology Program of Zhejiang Provincial Department of Transport (No. 20170426001).

\section{References}

1. Miettinen R., Paavola S. (2014) Beyond the BIM utopia: Approaches to the development and implementation of building information modeling. Automation in construction, 43: 84-91.

2. Lee N., Salama T., Wang G. (2014) Building information modeling for quality management in infrastructure construction projects. Computing in Civil and Building Engineering: 65-72.

3. Xihua D., Li F., Yang Y., Xian R. (2017) Building and Maintenance Integated BIM in Humen Second Bridge (in Chinese). China Highway: 68-71.
4. Yan Z., Chen H., Li F., Guo Y., Yan Y. (2017) Application of BIM technology in the Digital Steelwork Factory in Humen Second Bridge. Journal of Highway and Transportation Research and Development, 13: 239-40. 